\title{
Duplex Sonographic Evaluation of Hepatic Vasculature in Cirrhosis
}

\author{
Ahmad K, ${ }^{1}$ Khanna SK, ${ }^{1}$ Sundas $A,{ }^{1}$ Rauniyar RK, ${ }^{1}$ Koirala $R,{ }^{1}$ Gupta $M K^{1}$ \\ ${ }^{1}$ Department of Radiodiagnosis, B.P. Koirala Institute of Health Sciences, Dharan, Nepal.
}

\section{* Corresponding Author:}

Dr. Kaleem Ahmad, Associate Professor,

Department of Radiodiagnosis,

B.P. Koirala Institute of Health Sciences, Dharan, Nepal.

Email: drkalim17@yahoo.co.in

\section{Citation}

Ahmad K, Khanna SK, Sundas A, Rauniyar RK, Koirala $R$, Gupta MK. Duplex Sonographic Evaluation of Hepatic Vasculature in Cirrhosis. Nepal Journal of Medical Sciences. 2013;2(1):13-9.

\begin{abstract}
Background: Cirrhosis of liver in an ancient illness, known to the mankind since antiquity. Alcohol consumption is the most important cause of micronodular cirrhosis and chronic hepatitis is the most frequent cause of macronodular cirrhosis. The objective of this study was to assess the morphological changes and flow haemodynamics in portal vein, hepatic vein and hepatic artery using various Doppler parameters.
\end{abstract}

Methods: The study was done on prospective basis in 35 patients with clinical diagnosis of cirrhosis and these patients were subjected to ultrasonography of the abdomen for the assessment of morphological changes and flow haemodynamics in hepatic vasculature.

Results: Portal venous blood flow becomes reversed with advanced portal hypertension, veno-occlusive disease and portosystemic shunts. Reduced portal blood flow velocity was observed in majority of the patients and was found to be lower in patients with history of variceal haemorrhage. Hepatic artery resistive index was found to be significantly higher in patients with dilated coronary veins as compared to those with normal non-dilated veins. Dilated paraumbilical vein was the most consistently observed collateral followed by dilated coronary veins. Abnormal hepatic vein flow profiles are seen in patients with cirrhosis, with decreased amplitude of phasic occilation pattern being the most frequently observed abnormality.

Conclusion: Although many factors may affect the accuracy of volume flow and velocity measurements and the flow profile of the liver vasculature may change in different situations, Doppler ultrasound is useful in the assessment of the patient with cirrhosis and portal hypertension.

Keywords: Duplex sonography; cirrhosis; hepatitis; portal vein hypertension; collaterals

\section{Background:}

A ccording to the World Health Organization (WHO) cirrhosis is defined as diffuse process characterized by fibrosis and the conversion of normal liver architecture into structurally abnormal nodules. ${ }^{1}$ Cirrhosis is the combination of cellular necrosis, fibrosis and nodular regeneration and the symptoms are due to the effects of these processes, which occur in various degrees. ${ }^{2}$ Cirrhosis has been classified as micronodular in which nodules are less than $3 \mathrm{~mm}$ in diameter and macronodular characterized by nodules of varying size more than $3 \mathrm{~mm} .^{3}$ Alcohol consumption is the 
most important cause of micronodular cirrhosis and chronic viral hepatitis is the most frequent cause of macronodular cirrhosis. ${ }^{3}$ Other etiologies are biliary cirrhosis (primary and secondary), Wilson's disease, cryptogenic, Indian childhood cirrhosis, hemochromatosis, thallasemia, alpha1-antitrypsin deficiency, galactosemia, type IV glycogen storage disease, tyrosinosis and fructose intolerance etc. Portal hypertension is a major complication of cirrhosis in which the patient may present with haemorrhage from gastroesophageal varices, splenomegaly, ascites, acute and chronic hepatic encephalopathy.

Color Doppler and spectral Doppler ultrasound is noninvasive, easily available and cheap modality and it plays an important role in the study of the hepatic vasculature and the portal venous system. Colour Doppler imaging promptly identifies the portal vein, the hepatic artery and the hepatic vein and can confirm their patency and flow direction. In patients with hepatic cirrhosis, Doppler ultrasound is the initial technique for confirming suspected portal hypertension.

The purpose of this study was to determine the morphological changes in hepatic vasculature and the flow hemodynamics in portal vein, hepatic vein, hepatic artery using various Doppler parameters in cases of cirrhosis.

\section{Methods:}

This was a prospective study conducted in 35 patients with cirrhosis in Department of Radiodiagnosis, B. P. Koirala Institute of Health Sciences, Nepal over a period of one year. The study was approved by the institute and written informed consent was obtained from all the patients. Cirrhosis was diagnosed in patients of chronic liver disease on the basis of certain clinical, biochemical and Ultrasonography (USG). All the patients were subjected to USG of abdomen after overnight fasting of 8-10 hours.

Both gray scale and Doppler imaging were performed with 3.5 MHz curvilinear phased array transducer on SIEMENS G-50 ultrasound machine. Initially gray scale examination was performed to visualize the liver echotexture and nodularity. A 7.5 MHz linear array transducer was also used for the surface nodularity wherever required. The spleen was measured along its long axis with the left side of the patient elevated. Preliminary gray scale examination was also performed to seek for collaterals and free fluid in the peritoneal cavity.

Liver was imaged with the patient in the supine and right anterior oblique positions with subcostal acoustic window and intercostal scans. Longitudinal scans of the liver were obtained in midclavicular line and midline, measuring the longitudinal diameters of the right and left lobes respectively. For measuring Caudate lobe to Right lobe ratio $(\mathrm{C} / \mathrm{RL})$, transverse sonogram of the liver was obtained at the bifurcation of the main portal vein using the main portal vein as demarcating landmark. Later Doppler sonography was performed; the vessels were examined with curvilinear phased array transducer. The entire length of the portal vein was examined from an anterior abdominal subcostal approach, using a right paramedian, slightly oblique plane. From the hilum to the intrahepatic bifurcation, the portal vein was imaged, right and left portal branches were visualized with an oblique scan. Maximum portal blood flow velocity (PBFV) and the direction of the blood flow were recorded. In all the cases, the caliber of the portal vein was measured during deep inspiration around the middle of the portal trunk and again at the end of expiration and variation in the caliber was noted.

The splenic vein was examined at the splenic hilum and the remainder of the splenic vein to its confluence with the superior mesenteric vein was examined in transverse sections. The hepatic venous system was best visualized with the patient supine or turned slightly to the left at the end of deep inspiration. The morphologic appearance of the hepatic veins was evaluated by assessing caliber and course. Doppler study was then performed by positioning the sample volume cursor within the hepatic vein at a distance of 3-6 cm from the outlet of the vessel into the inferior venacava. The waveform characteristics were recorded. The hepatic artery was insonated at its origin at the celiac axis and where it crosses the portal vein. The Doppler tracings from the hepatic artery were obtained and the resistivity index (RI) and pulsatility index (PI) were calculated.

To demonstrate the paraumbilical vein, the ligamentum teres was used as a landmark and the presence of a patent paraumbilical vein was verified by color Doppler. The coronary vein was identified in the plane of aorta, the maximum luminal diameter of the coronary vein was measured in longitudinal plane and the direction of the blood flow within it assessed. Splenorenal collaterals were identified at the lower pole of spleen as tortuous vessels within a high velocity Doppler signal. The short gastric veins appearing around the splenic hilum were detected by scanning in the eighth or ninth left intercostal spaces. After data collection, the findings were tabulated and subjected to statistical analysis. 


\section{Results:}

Total 35 cases were included in the study, the age of the patients varied from 18-77 years. Maximum number of cases, i.e. $12(34 \%)$ were observed in the age group of 41-50 years while minimum number of case was in the $<20$ years age group (3\%). Among these, 20 were males and 15 were females with preponderance of males over females.

The most frequent clinical feature was abdominal distension which was observed in 31 patients $(89 \%)$ followed by jaundice in 20 patients (57\%), upper gastrointestinal hemorrhage in 11 patients (31\%) and 7 $(20 \%)$ patients had features of hepatic encephalopathy. On physical examination, 24 patients presented with ascites, abdominal distension, splenomegaly, hepatomegaly and dilated abdominal veins (Table 1).

Table 1: showing clinical features in liver cirrhosis.

\begin{tabular}{lcc}
\hline \multicolumn{1}{c}{ Clinical Features } & $\begin{array}{c}\text { No. of } \\
\text { Patients }\end{array}$ & Percentage (\%) \\
\hline Ascites & 24 & 69 \\
$\begin{array}{l}\text { Jaundice } \\
\begin{array}{l}\text { Gastrointestinal } \\
\text { haemorrhage } \\
\text { Hepaticencephalopathy }\end{array}\end{array}$ & 7 & 57 \\
$\begin{array}{l}\text { Palpable liver } \\
\text { Palpable spleen }\end{array}$ & 13 & 31 \\
Dilatedabdominalveins & 8 & 20 \\
\hline
\end{tabular}

Laboratory examination revealed, increased total serum bilirubin level in 18 patients. The value of Serum Aspartate Transaminase (AST) was found much deranged as compared to Serum Alanine Transaminase (ALT). Serum Alkaline Phosphatase level showed mild derangement in majority of patients.

Grey scale sonography of the liver revealed Caudate Lobe / Right Lobe ratio (C/RL) greater than 0.65 in 16 patients $(46 \%)$. The ratio was less than or equal to 0.65 in 19 patients $(54 \%)$. Deranged echotexture of liver was seen in 21 patients $(60 \%)$, surface nodularity (Figure1) of liver in 26 patients $(74 \%)$, splenomegaly in 20 patients $(57 \%)$, ascites in 31 patients $(89 \%)$. Of the 31 patients in whom ascites was observed on sonography, 24 (69\%) had clinically detectable ascites while in other 7 , it could not be detected clinically (Table 2).

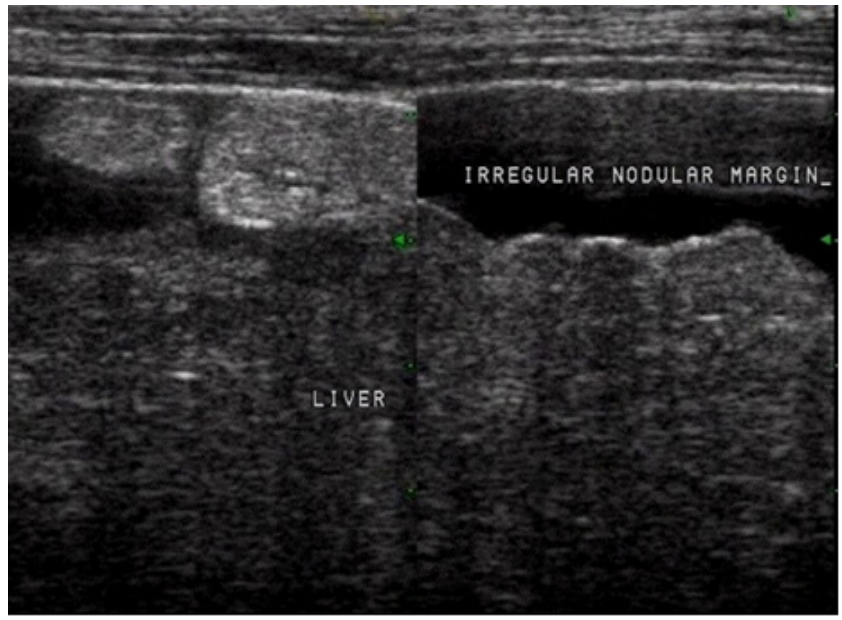

Figure 1: Gray scale ultrasound showing nodular hepatic surface.

Table 2: Distribution of cases according to ultrasonographic appearance of liver

\begin{tabular}{lcccccc}
\hline Group & \multicolumn{2}{c}{ C/RL Ratio } & \multicolumn{2}{c}{ Echotexture } & \multicolumn{2}{c}{$\begin{array}{c}\text { Surface } \\
\text { nodularity }\end{array}$} \\
& $>0.65$ & $£ 0.65$ & Normal & Altered Present Absent \\
\hline Males & 10 & 10 & 6 & 14 & 16 & 4 \\
Females & 6 & 9 & 8 & 7 & 10 & 5 \\
Total & 16 & 19 & 14 & 21 & 26 & 9 \\
\hline
\end{tabular}

On Doppler sonography, main portal vein was seen in all the 35 patients. Dilatation of the portal trunk of more than $13 \mathrm{~mm}$ was found in 20 patients $(57 \%)$. In 8 of these cases $(40 \%)$ the caliber of the vessel was greater than $15 \mathrm{~mm}$. In 15 out of 35 cases $(43 \%)$ the caliber of the portal vein was found to be less than or equal to $13 \mathrm{~mm}$. The lack of normal caliber variation during deep inspiration was demonstrated in the main portal vein (Figure 2) and the splenic vein. Lack of normal ( $>20 \%$ ) caliber variation in the main portal vein was observed in 31 patients (89\%) (Table 3 ).

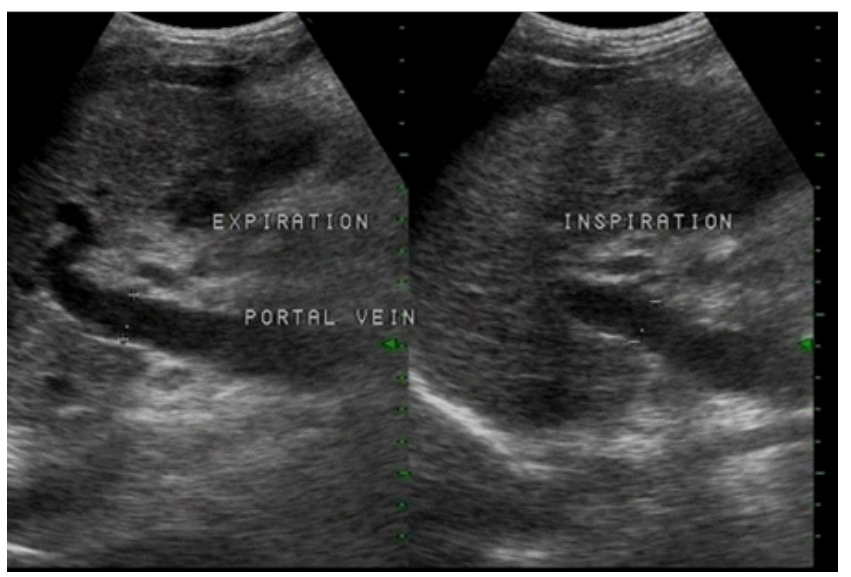

Figure 2: Sonogram showing dilated main portal vein with decreased phasic variation. 
Table 3: portal vein diameter and variations in the caliber of main portal vein

\begin{tabular}{lcccc}
\hline & MPV Diameter $(\mathrm{mm})$ & \multicolumn{2}{c}{ Phasic Variation } \\
Group & $£ 13$ & $>13$ & $<20 \%$ & $>20 \%$ \\
& 8 & 12 & 18 & 2 \\
Males & 7 & 8 & 13 & 2 \\
Females & 15 & 20 & 31 & 4 \\
Total & & & & \\
\hline
\end{tabular}

Dilatation of the splenic vein $(>10 \mathrm{~mm})$ was observed in 22 of 35 patients $(63 \%)$ and the lack of normal caliber variation in the splenic vein was observed in 28 patients $(80 \%)$.

The average value of PBFV was found to be $12.56 \pm 1.7 \mathrm{~cm} /$ sec. 11 patients had history of variceal hemorrhage, in which 8 patients had $\mathrm{PBFV}<13 \mathrm{~cm} / \mathrm{sec}, 1$ patient had $\mathrm{PBFV}$ $>15 \mathrm{~cm} / \mathrm{sec}$ and in the rest 2 patients it lied between $13-15$ $\mathrm{cm} / \mathrm{sec}$.

Abnormal flow patterns in portal vein were encountered in 10 of 35 patients (29\%). Normal hepatopedal flow was observed in 25 patients. Reversed, hepatofugal flow was the most frequent abnormality recorded, seen in 8 out of 10 patients. Two patients with splenorenal collaterals had reversed flow in four vessels: the right, left, main portal vein and splenic vein (Figures 3 and 4).

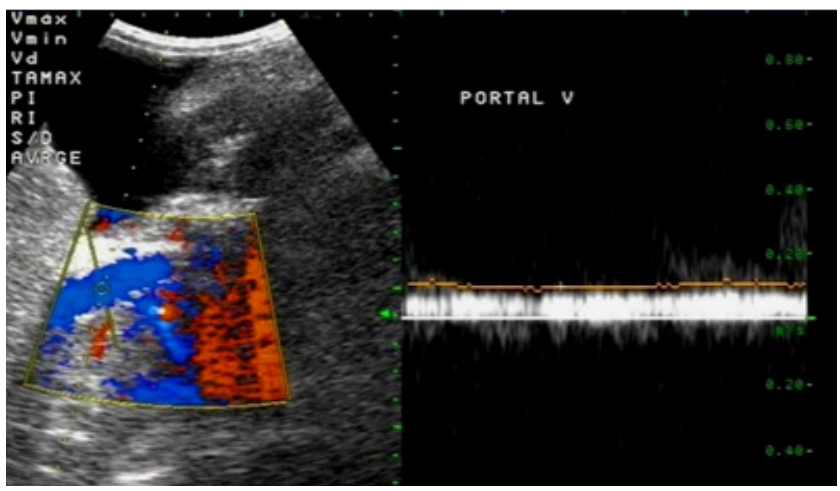

Figure 3: Doppler study of the main portal vein showing decrease blood flow velocity with hepatofugal flow.

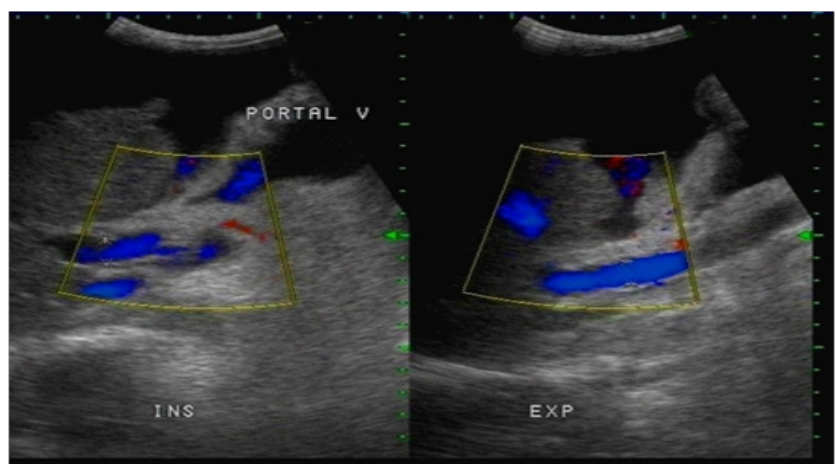

Figure 4: Color Doppler sonogram showing hepatofugal flow in the main portal vein.
Abnormal hepatic vein waveform (Figure 5) was detected in 17 patients (49\%). Decreased amplitude of the phasic oscillation (HV1 pattern) was observed in 10 of these patients (10/35). Completely flattened waveform without any phasic oscillation, the HV2 pattern was demonstrated in 7 cases (7/35. Doppler tracings of 18 out of $35(51 \%)$ showed normal triphasic waveform i.e. HV0 pattern. No attempt was made to relate the hepatic vein waveform with severity of the disease (Table 4).

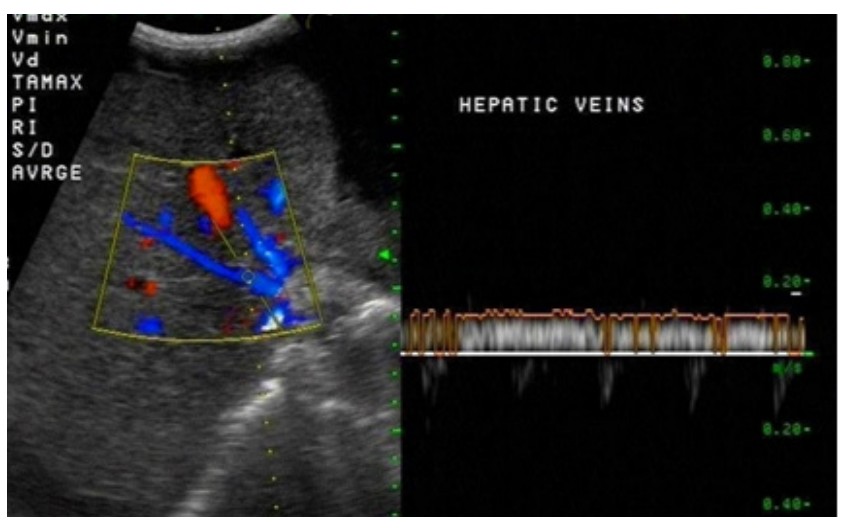

Figure 5: Doppler waveform of the hepatic vein showing reduction in amplitude and flattened waveform.

Table 4: Abnormalities of Hepatic Vein Waveform (HVHepatic vein).

\begin{tabular}{cccc}
\hline \multirow{2}{*}{ Group } & \multicolumn{3}{c}{ Waveform Patterns } \\
& HV0 & HV1 & HV2 \\
\hline Males & 12 & 6 & 2 \\
Females & 6 & 4 & 5 \\
Total & 18 & 10 & 7 \\
\hline
\end{tabular}

Hepatic arterial resistive indices in 20 out of 35 patients had RI value in the range 0.6-0.7. The mean hepatic artery RI for all the 35 patients was $0.74 \pm 0.11$. The hepatic artery RI was found to be higher in patients with dilated coronary veins compared with those with normal non dilated veins (Table 5).

Table 5: Hepatic artery RI in Patients with and without Dilated Coronary Veins.

\begin{tabular}{lcc}
\hline Group & $\begin{array}{c}\text { No of patients with Hepatic } \\
\text { artery RI in the range }\end{array}$ & $\begin{array}{c}\text { Mean } \\
\text { Hepatic }\end{array}$ \\
& $0.50-0.60 \quad 0.60-0.70 \quad 0.70-0.80$ & Artery RI \\
\hline
\end{tabular}

\section{Patients}

withoutdilated

20

10

$0.74 \pm 0.11$

coronaryveins

Patients

with dilated

coronaryveins 
Portosystemic collateral veins were detected in 16 patients $(46 \%)$. Dilated paraumbilical vein $(>3 \mathrm{~mm}$ ) (Figure 6) was the most frequently observed collateral (10 patients; 29\%), superficial abdominal varices extending caudally from dilated paraumbilical veins in 6 of these 10 patients, dilated coronary veins $(>6 \mathrm{~mm})$ in 8 patients $(23 \%)$, splenorenal and short gastric collaterals (Figure 7) in 4 patients (12\%), gastrorenal collaterals in 2 patients $(6 \%)$ and one patient showed the presence of retroperitoneal collaterals (3\%).

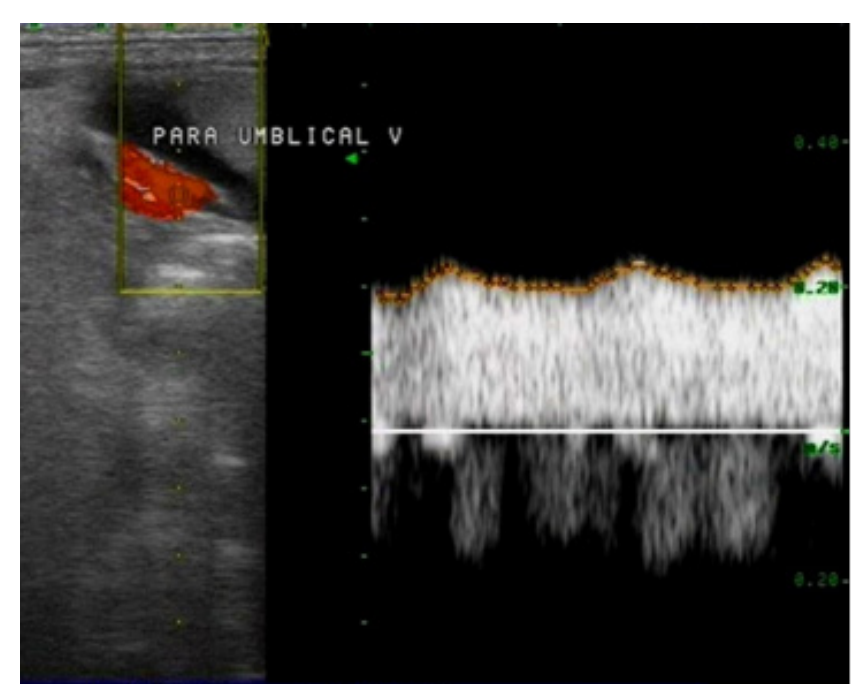

Figure 6: Color Doppler image demonstrating paraumbilical collateral in the ligamentum teres.

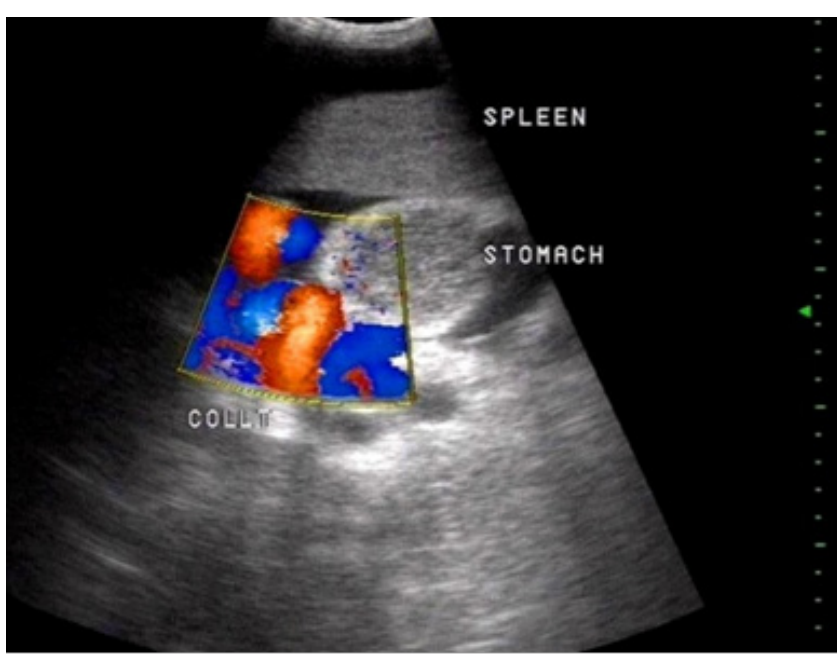

Figure 7: Color Doppler showing collateral vessels between upper pole of spleen and stomach consistent with short gastric collaterals.

\section{Discussion:}

Color and spectral Doppler ultrasound plays an important role in the study of the hepatic vasculature and the portal venous system and is safe, non-invasive, easily available modality used as the initial technique for confirming suspected portal hypertension in patients of hepatic cirrhosis.
Bolondi et al. $^{4}$ examined 160 patients with portal hypertension and dilatation of the portal vein of more than $1.3 \mathrm{~cm}$ was detected in 73 of 129 patients, dilatation of the splenic vein of more than $10 \mathrm{~mm}$ in $70 \%$ of patients and also demonstrated disappearance of normal caliber variation $(>20 \%)$ during respiration in splenic or superior mesenteric veins. In the present study, dilatation of the portal trunk of more than $13 \mathrm{~mm}$ was found in 20 patients, dilatation of the splenic vein was observed in $63 \%$ of patients, lack of normal $(>20 \%)$ caliber variation in the main portal vein was observed in 31 out of 35 patients and the disappearance of normal $(>20 \%)$ caliber variation in the splenic vein was observed in 28 out of 35 patients. The observations in our study are comparable to the work done by the previous observers.

Study done by Tomic et al..$^{5}$ emphasized that portal blood flow velocity decreases in portal hypertension and the PBFV was $9.38 \mathrm{~cm} / \mathrm{sec}$ in portal vein and $10.17 \mathrm{~cm} / \mathrm{sec}$ in splenic vein and concluded that decrease of the PBFV below $12 \mathrm{~cm} /$ sec is a reliable indicator of the portal hypertension. In our study, the average value of PBFV was found to be $12.56 \mathrm{~cm} /$ $\sec \pm 0.11 \mathrm{~cm} / \mathrm{sec}$, which is consistent with the studies of previous researchers. ${ }^{8,12-15}$

Ralls $^{6}$ evaluated 147 patients with diffuse liver disease; abnormal flow pattern was detected in $25 \%$ of which reversed flow was the most frequent abnormality occurring in $19 \%$ of patients. In our study abnormal flow patterns were encountered in 10 patients (29\%), reversed (hepatofugal) flow in 8 patients $(23 \%)$ and 2 patients with splenorenal collaterals had reversed flow. The observations in the present study are somewhat consistent with the previous observers. ${ }^{6,16-18}$

Bolondi et al. ${ }^{7}$ classified waveform characteristics of hepatic veins into 3 groups, HV0, HV1, and HV2 (HV-Hepatic veins). Among cirrhotic patients, HV0 was found in (50\%), HV1 in $(32 \%)$ and HV2 in $11(18 \%)$. Thus considering the HV0 pattern as the normal Doppler waveform, abnormalities in the waveform of hepatic veins, occurred in $50 \%$ of cirrhotic patients. In our study of 35 patients with cirrhosis, we discovered abnormal hepatic vein waveform in 17 patients. Decreased amplitude of the phasic oscillations (HV1 pattern) was observed in 10 patients while completely flat waveform without any phasic oscillation (HV2 pattern) was demonstrated in 7 patients. The rest 18 patients showed normal triphasic waveforms. Our findings are more in conformity with the observations of Bolondi et al. ${ }^{7}$

Vassiliades et $\mathrm{al}^{8}$ performed Doppler sonography in 43 
patients of diffuse liver disease to determine hepatic arterial resistive index. Hepatic artery RIs ranged from $0.64 \pm 0.06$ in patients with early cirrhosis to $0.68 \pm 0.09$ in patients with severe inflammation. They were unable to demonstrate any correlation between the RI value and the degree of hepatic pathology. In the present study, a dilated tortuous hepatic artery was seen in 6 patients. Hepatic arterial resistive indices especially RI were measured in all the 35 cases. Since no control was taken in our study, the study group was split into several sub groups and the hepatic arterial RI was measured in these sub groups, the mean hepatic arterial RI was found to be $0.74 \pm 0.11$. Thus the observations in the present study are consistent with the findings of previous researchers.

Ralls $^{6}$ in his study detected collateral portal veins in which paraumbilical veins with superficial abdominal varices were the commonest. Sacerdoti et al. ${ }^{9}$ observed a patent paraumbilical vein in $34 \%$ of patients. In our study portosystemic collaterals were detected in 16 patients (46\%); dilated paraumbilical vein was the most frequently observed collateral (10 patients), superficial abdominal varices in 6 of these 10 patients, dilated coronary veins in 8 patients, splenorenal collaterals in 4 cases, gastrorenal collaterals in 2 cases and one patient showed the presence of retroperitoneal collaterals. A patent paraumbilical vein was the most frequently observed collateral, which is consistent with the observations of Ralls ${ }^{6}$ and Sacerdoti ${ }^{9}$.

Iwao et al. ${ }^{10}$ calculated liver vascular index as the ratio of the portal venous velocity to hepatic arterial pulsatility index. For the diagnosis of liver cirrhosis or portal hypertension the best cut-off value of the liver vascular index was suggested as $12 \mathrm{~cm} / \mathrm{s}$ with a sensitivity and specificity of $97 \%$ and $93 \%$ respectively. In our study the vascular index was found to be below $12 \mathrm{~cm} / \mathrm{s}$ in majority of the patients $(32 / 35$. This is in accordance with the findings of Iwao et al. ${ }^{10}$

\section{Conclusion:}

Ultrasound is the non-invasive diagnostic modality which aids in long-term management of cirrhotic patients. Morphological changes such as volume redistribution, surface nodularity, parenchymal nodularity and echotexture can be demonstrated on grey scale Ultrasonography. Color Doppler is an excellent modality for delineating the complex hemodynamics of portal hypertension in patients of cirrhosis. Detection of portosystemic collaterals is beneficial in the management of esophagogastric varices and portosystemic encephalopathy. Assessment of hepatic and portal veins, hepatic artery and varices are better evaluated on Doppler sonography in cirrhosis.

Conflict of interest: None disclosed

\section{References:}

1. Anthony PP, Ishak KG, Nayak $\mathrm{NC}$, et al. The morphology of cirrhosis: definition, nomenclature, and classification. Bull World Health Organ 1977;55:52140.

2. Stone WD. Cirrhosis of Liver. Practitioner 1973;210:612-7.

3. Brown JJ, Naylor MJ, Yagan N. Imaging of hepatic cirrhosis. Radiology 1997;202:1-16.

4. Bolondi L, Mazziotti A, Arienti V, et al. Ultrasonographic study of portal venous system in portal hypertension and after portosystemic shunt operation. Surgery 1984;95:261-9.

5. Tomic D, Jesic R, Krstic, et al. Drop of the portal blood flow: An important sign of the portal hypertension. Arch Gastroenterohepatol 2001;20:89-92.

6. Ralls PW. Colour Doppler Sonography of the hepatic artery and portal venous system. Am J Roentgenol 1990;155:517-25.

7. Bolondi L, LiBaasi S, Gaiani S, et al. Liver cirrhosis: Changes of Doppler waveform of hepatic veins. Radiology 1991;178:513.

8. Vassiliades VG, Ostrow TD, Chezmar JL, et al. Hepatic arterial resistive indices: correlation with the severity of cirrhosis. Abdom Imaging 1993;18:61-5.

9. Sacerdoti D, Merkel C, Bolognesi M, et al. Hepatic arterial resistance in cirrhosis with and without portal vein thrombosis: relationship with portal hemodynamics. Gastroenterolgy 1995;108:1152-8.

10. Iwao T, Tyonaga A, Kazuhiko O, et al. Value of Doppler ultrasound parameters of portal vein and hepatic artery in the diagnosis of cirrhosis and portal hypertension. Am J Gasteroenterol 1997; 92:1012-7.

11. Gorka W, Kagalwalla A, Mc Parland Bj, et al. Diagnostic value of Doppler ultrasound in the assessment of liver cirrhosis in children: histopathological correlation. J Clin Ultrasound 1996;24:287-95.

12. Zironi G, Gaiani S, Fenyves D, et al. Value of measurement of mean portal flow velocity by Doppler flowmetry in the diagnosis of portal hypertension. $\mathrm{J}$ 
Hepatol 1992;16:298-303.

13. Ohnishi K, Saito M, Nakayama T, et al. Portal venous hemodynamic in chronic liver disease: effect of posture change and exercise. Radiology 1985;155:757-61.

14. Ohnishi K, Saito M, Sato S, et al. Portal hemodynamics in idiopathic hypertension (Banti's syndrome). Gastroenterology 1987;92:751-8.

15. Moriyasu F, Nishida O, Ban N, et al. Measurement of portal vascular resistance in patients with portal hypertension. Gastroenterol 1986;90:710-7.
16. Burcharth F, Aagaard J. Total hepatofugal portal flow in cirrhosis demonstrated by hepatic portography. Rofo 1988;148:47-9.

17. Kawasaki T, Moriyasu F, Nishida O, et al. Analysis of hepatofugal flow in portal venous system using ultrasonic Doppler duplex system. Am J Gastroenterol 1989;84:937-41.

18. Gaiani S, Bolondi L, Li Bassi S, et al. Prevalence of spontaneous hepatofugal flow in liver cirrhosis. Clinical and endoscopic correlation in 228 patients. Gastroenterology 1991;100:160-7. 